\title{
Design of Interactive Aids for Children's Teeth Cleaning Habits
}

\author{
Cheng Chuko, Fang-Lin Chao*, Hsin-Yu Tsai \\ Department of Industrial Design, Chaoyang University of Technology, Taichung, 436, Taiwan, R.O.C.
}

\author{
A R T I C L E I N F O \\ Article history: \\ Received: 09 February, 2020 \\ Accepted: 25 March, 2020 \\ Online: 04 April, 2020
}

Keywords:
Product design
Arduino
Teeth cleaning
Interactive aids

\section{Introduction}

The most common dental problem of concern in children is dental caries. Dental plaque is considered a possible causative agent of major dental diseases. Oral health affects chewing, nutrition, speaking, socializing. As a result, people must develop oral care skills at an early age. The implementation of oral disease prevention includes education and the integration of family support. This design study aims to assist parents and to reduce children's fear while cleaning their teeth.

\subsection{Oral Health Education}

Advocacy and family coordination are vital for implementing oral health education. Children are often unfamiliar with initial skills. A survey of Taiwan's 3-6 year-olds showed that the average permanent dental caries experience index (DMFT) of school children was 3.31. This index indicates the need for promoting children's oral health programs. Taiwanese children prefer sugary drinks, which have a high correlation with severe dental caries. The frequency of sugary drinks for children in Taiwan is 4-6 times a week. Therefore, it is necessary to implement oral health education and implement correct dental cleaning habits from early childhood.

Good oral hygiene is dependent upon the effectiveness and the ease with which the procedure s carried out. The most common preventive dental program is the modified Bass approach [1] (Figure 1). Domestic academics and American public health

\footnotetext{
*Fang-Lin Chao, Email: flin@ cyut.edu.tw
}

scholars also recommend the Bass method for its high efficiency and "horizontal short-distance movements." Its main points are:

(1) Place the toothbrush at a 45-degree angle on the gum line.

(2) Brush using a gentle circular motion: downward for the upper teeth and upward for the lower teeth, and brush 2 3 teeth at a time.

(3) Use the same procedure for the lower teeth, except rotating in an upward direction toward the medial teeth.

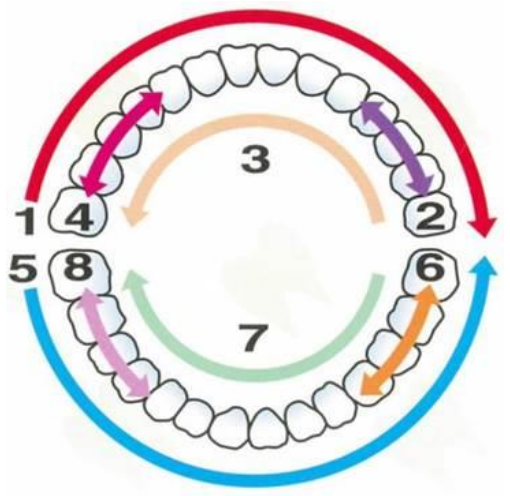

Figure 1. Brushing sequence of the Bass method.

A reduction of interdental plaque and gingival inflammation requires a suitable brushing technique. Poyato-Ferrera et al. [2] found that the modified Bass method is superior for supragingival 494 


\section{Chuko et al. / Advances in Science, Technology and Engineering Systems Journal Vol. 5, No. 2, 494-499 (2020)}

plaque removal. Studies also show that the modified Bass technique was useful for children $[3,4]$. The Scrub and Bass methods (with flossing) are effective in eliminating or reducing plaque and gingivitis in 11-year-old children using a junior toothbrush.

\subsection{Analysis of Existing Products}

Extant products seek to attract young children with appealing anthropomorphic images. It is difficult for purely informational products that lack substantial interaction to keep young children engaged for a long time (Figure 2). These textbooks are like a toy and cannot be interacted with in person; so it is not easy to raise the expectation of continuous exploration.

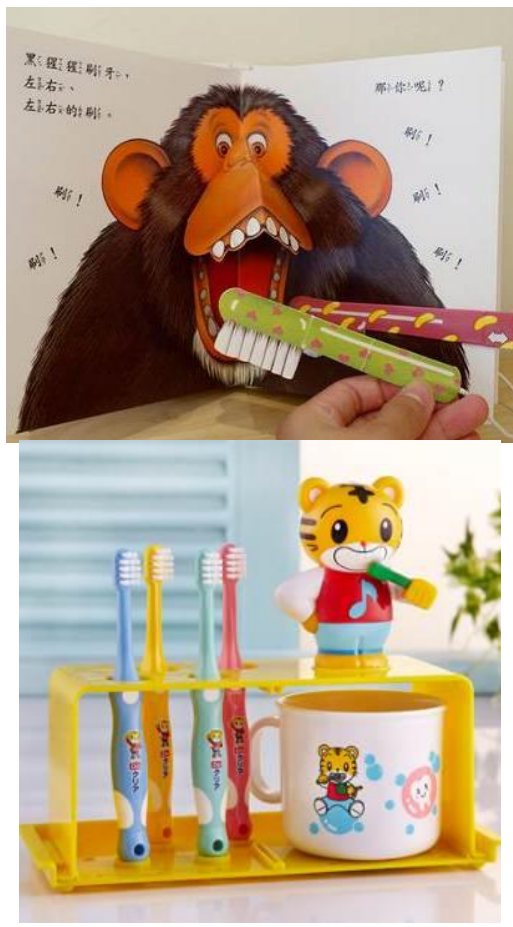

Figure 2. Interactive dental cleaning products (a) gamebook, (b) music-induced voice cleaning.

Philips Sonicare marketed by Oral Healthcare (Figure 3) is a companion product for teeth cleaning that is currently on the market. Power toothbrushes remove plaque more effectively by brush head motion. For example, Sonicare claims to remove plaque as a result of dynamic fluid activity [5]. Studies show that the use of a Sonicare Airfloss results in less removal of tooth enamel when compared to the use of a standard toothbrush [6]. A reduction in plaque in the posterior and hard-to-reach areas indicated the benefit of the design [7].

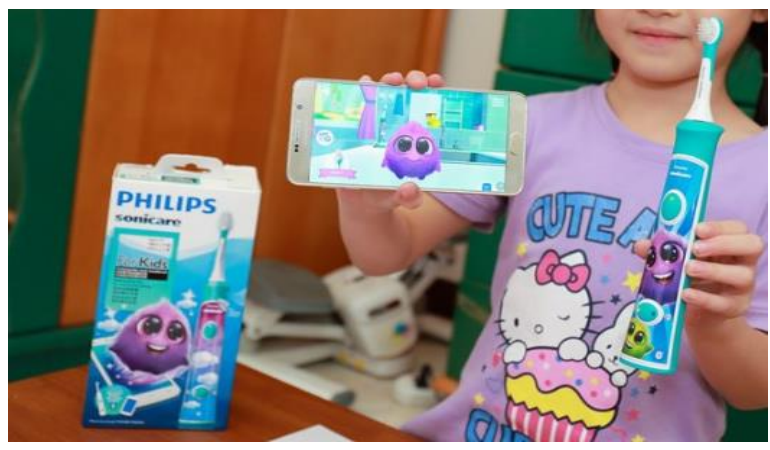

Figure 3. Sonicare children's toothbrush.
Sonicare features include timed tooth cleaning, an app music reminder to change the part of the mouth being brushed every 30 seconds, and a link with an interactive game app. Its advantage is flexible content; however, the user must be familiar with smartphone operation. Another study [8] identified several other oral health-focused apps in the Apple App Store and Google Play. Apps identified using the following search phrases: oral health, dental health, teeth health, tooth health, dental care, and oral care. However, the quality of the reviewed apps was generally poor [9]. Apps need theoretically grounded content and adherence to ethical design principles for persuasive health technologies. The Oral-B and Sonicare apps can connect with a phone via Bluetooth. However, those apps performed poorly on standard usability heuristics [10].

\section{Survey and Analysis}

First, we surveyed the toothbrushing habits of children to find suitable entry points for product design. Items included current brushing status, the attitudes of children and parents toward tooth brushing, and methods of parental assistance in building dental cleaning habits.

\subsection{Method}

A total of 204 families with schoolchildren in the age range of 3-6 years old were selected in a randomized controlled family survey. The inclusion criteria used to select the samples for the study were as follows: (i) healthy children between the age group; (ii) schoolchildren who live with their parents. The survey distributed through both paper and website.

\subsection{Results and Discussion}

According to the survey data, more than half of the families were double-income families, and only $40 \%$ of the carers were full-time caregivers. At present, more than $90 \%$ of the parents demonstrated an awareness of oral care. However, more than $70 \%$ of children needed to be accompanied by their parents when brushing their teeth. More than $70 \%$ of families reported purchasing education material or products for oral health care. 3.5 $\%$ families reported their child had tooth decay, and less than $20 \%$ of children demonstrated knowledge of preventative oral care.

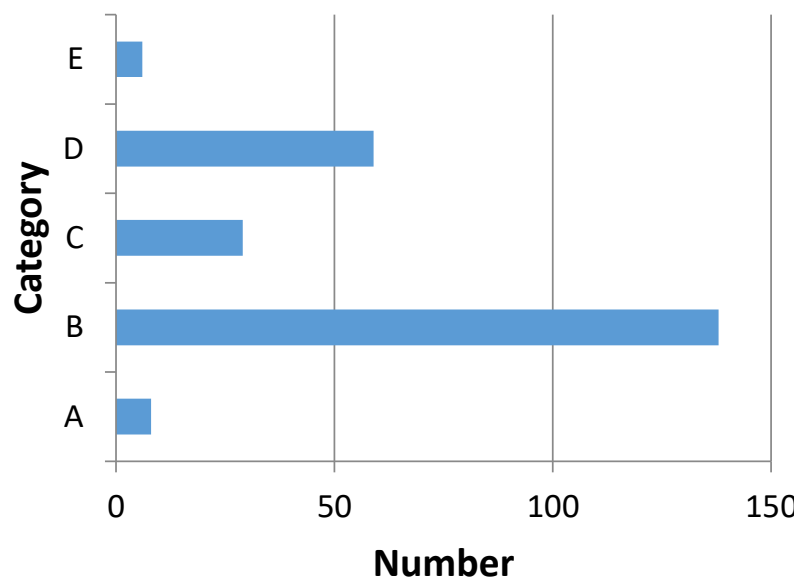

Figure 4. Children's brushing habits. 


\section{Chuko et al. / Advances in Science, Technology and Engineering Systems Journal Vol. 5, No. 2, 494-499 (2020)}

The Figure 4 shows the level of brushing reported by the families, as follows:

A: Child has not yet begun to brush their teeth.

B: The parent brushes child's teeth.

$\mathrm{C}$ : The child is self-motivated to brush teeth.

D: Parent motivates a child to brush teeth.

E: The child brushes teeth with parental assistance.

Items $\mathrm{B}, \mathrm{D}$, and $\mathrm{E}$ involved parental participation, so the children could not take the initiative to complete these tasks themselves. Therefore, an educational product that plays the role of a parent could hypothetically reduce the burden on parents. If the product is impressive, the design could improve children's motivation to brush their teeth and improve the effectiveness of tooth cleaning.

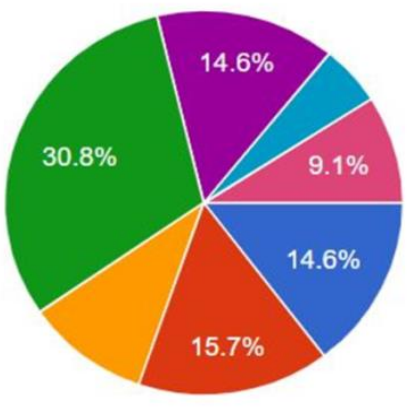

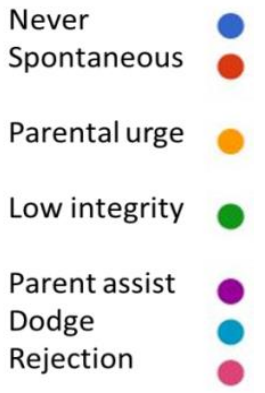

Figure 5. Children's behavior and attitude towards teeth cleaning

As shown in Figure 5, less than $20 \%$ of children can brush their teeth independently, and the rejection rate is quite high, at $15 \%$. Low quality meant that although the child brushed their teeth, they did not do so carefully, so they remained at risk for tooth decay. The survey results revealed the Bass method needs more promotion to families.

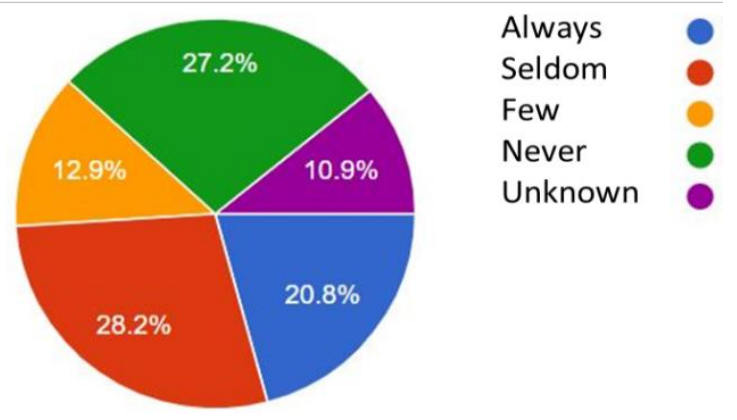

Figure 6. How often do children brush their teeth with the Bass method?

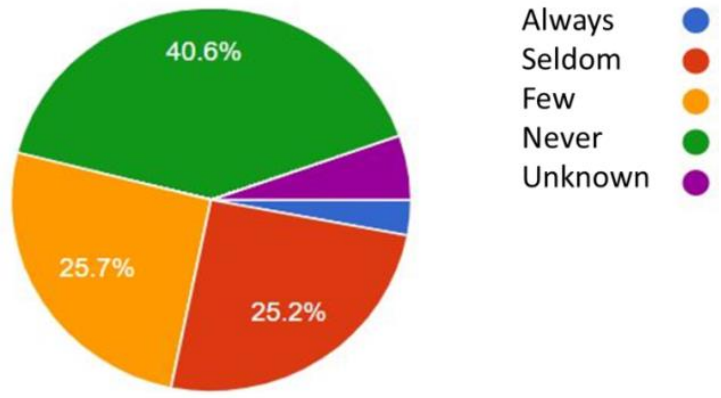

Figure 7. Completeness of Golden Teeth 333 code
As shown in Figure 6, parents report that only $21 \%$ of children can brush their teeth with the Bass method. Most children or parents are not familiar with the main points of the Bass method. The data also shows that there is a need to increase through auxiliary tools.

The "333 Code" refers a better way to brushing teeth: at least three times a day, brushing one's teeth within 3 minutes after a meal and brushing one's teeth for more than 3 minutes each time. Only less than $10 \%$ of people reported performing it correctly.

The Association of Dental Surgeons points out that the rate of tooth decay in children is high, with an average of five cases of tooth decay per child. The Department of Health appeals to all age groups, from infants, adolescents, and the elderly for independent health management.

The market survey found that most parents had an awareness of establishing healthy habits (Figure 7): $79.2 \%$ reported wanting to comply as much as possible, but also reported difficulties with implementation. Researchers hoped that assisted design could develop independent healthcare habits in children from an early age.

\section{Design Approaches}

Through the questionnaire survey, we understand that the current situation of children's learning is significantly different from our expectations. Through various design concepts, we proposed to increase children's attempts with interactivity, attract them to try and reduce the burden on parents. Through the sharing mechanism, excellent performance can be linked with parents' rewards to form a positive learning cycle.

\subsection{Problem Definition}

The target user age for this study was 3- to 6-year-old children. The goal was to remove children's fear of cleaning teeth and implement tooth cleaning habits without parental assistance. It is easier to develop a habit if the process is enjoyable. A suitable game design challenges children depending on their age and ability. Suitable game types for children include distinguishing color, number, and order, recognition, and memory. At the age of five, children have basic concepts of mathematics, association, and space. Simple vocabulary games can reduce the fear of tooth brushing in children and assist parents in encouraging children's oral health.

A bionic "dentist partner" draws children's gazes and reduces the rejection of oral cleaning appliances. Interaction and encouragement between parents and children allow children to develop habits. A visually appealing form supplemented by smart monitoring functions facilitates observation of cleaning status. A "dentist partner" game uses an LED screen to ensure that children are excited to complete the cleaning process using the Bass method.

It is not easy to cultivate oral cleaning habits at an early stage, and some children are repulsed by stimulation. Toy toothbrushes and electric toothbrushes often appeal to children with cute shapes, but parents' supervision is required to urge children to clean their teeth properly. Adding childlike connotations and introducing stories makes cleaning teeth more fun for children.

\subsection{Arduino and Control Circuit}

Arduino is a typical software tool for building a variety of devices with available peripherals, tools, and techniques. The 


\section{Chuko et al. / Advances in Science, Technology and Engineering Systems Journal Vol. 5, No. 2, 494-499 (2020)}

Arduino ecosystem includes LED, Servo motors and stepper motors, wireless connectivity, and the Internet of Things. The technical forum provides more prominently featured in the advanced projects. We utilized a 4-digit 7-segment display [12] with a common cathode and a digit display that turns on when the digit pin state is LOW. The lights are lit one by one in the order of the brush. The user needs to stay in this position until the light index changes. The status of the palm movements identified using IMU inertial sensor to read the accumulated number of brush. When the cumulative number of swipes exceeds a specified target, the light moved to the next position to lead children's interaction (Figure 8).

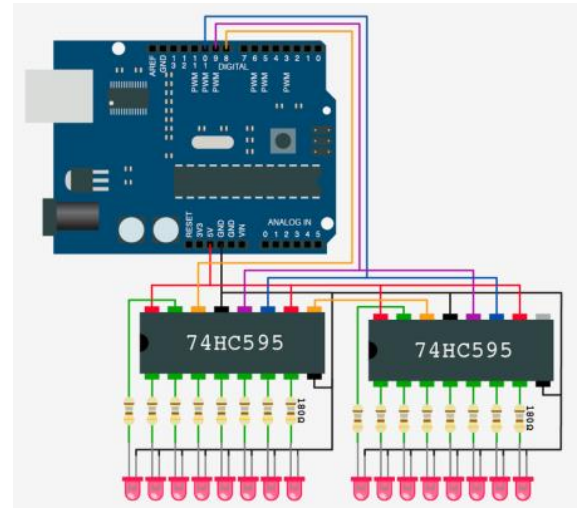

Figure 8. Hardware circuit. The device s comprised of an Arduino Nano microcontroller, MPR 121 Multiplexer, MPU 6050 IMU, and a Bluetooth [11]

\subsection{Product Design}

The initial concept of oral healthcare aid inspired by a whacka-mole game. Instead of striking a mole, the idea of the game was transformed into brushing specific teeth. The device design based on an anthropomorphic hippopotamus with an open mouth and exposed teeth. A capsule machine inside the device rewarded children with a surprise gift following tooth brushing (Figure 9). Children were trained in good tooth brushing habits through daily cleaning, with additional help from a parent app.

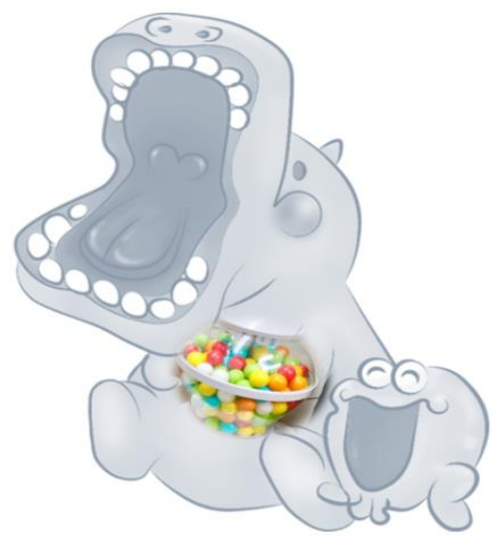

Figure 9. Final concept sketch

The model built by vacuum forming with a polyurethane mold. The buckled structure utilized to take into account the size of the body and the internal configuration (Figure 10). The appearance of the model set to a medium-sized doll, which intended to stimulate children's alertness and curiosity.

The chassis contained the dental cleaning indicator and timing function and could transmit the cleaning record, time spent brushing, and conditions to a parent's mobile phone. The hipposhaped teeth had indicator lights to indicate a brushing location. An Arduino program controlled the LED. Each tooth lit up sequentially according to Bass method and sensed whether there was brushing at the corresponding position. The toothbrush handle equipped with an electronic induction device, a Bluetooth module, and a battery, and the indicator light indicates the position of the brush; the inertial sensor [13] on the toothbrush handle detects whether the toothbrush is shaking to confirm that the child is brushing correctly. The counter and timer of the system can accumulate the number and time to identify the brushing situation.

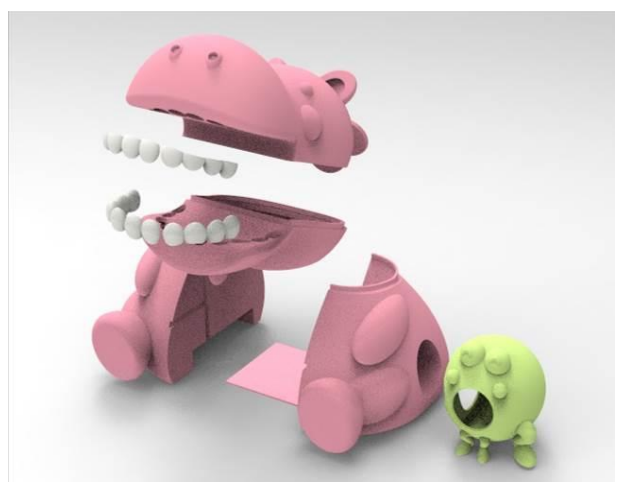

Figure 10. Assembly drawing of main accessories and appearance.

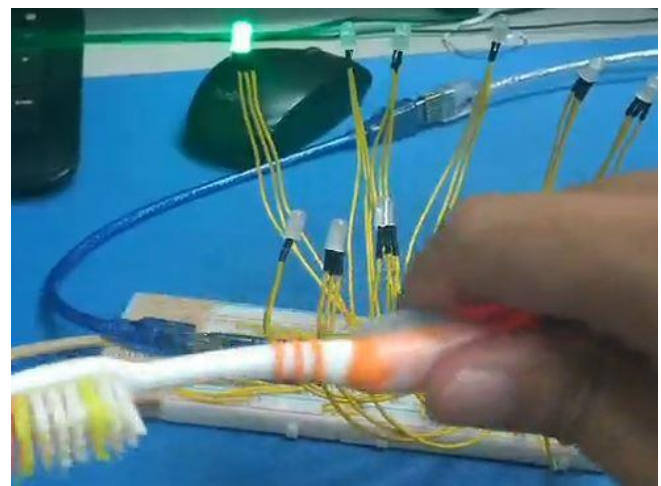

Figure 11. Sensors and LED indication configuration and the brush with an inertial sensor that wirelessly connected with the main circuit board.

Specifications of internal parts were as follows: Arduino Nano, HC-06 Bluetooth module, 74594 SIPO shift register, R.T.C. clock,

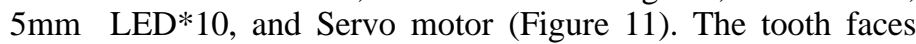
indicated with different colors: green for the external surface, blue for occlusal surface, and red for the internal surface.

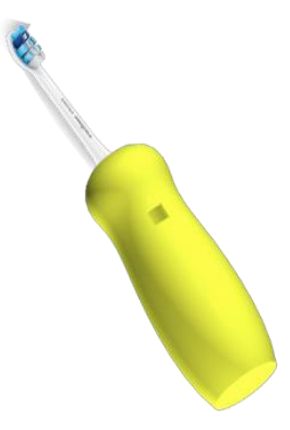

(a) (b)

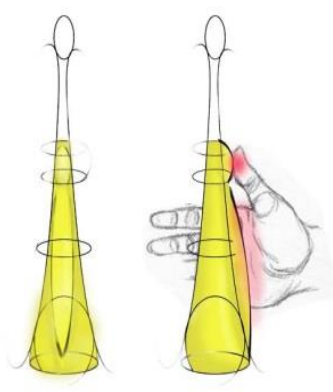

Figure 12. The rendering of toothbrush and (a) actual installation appearance, and (b) grip shape of a possible design. 


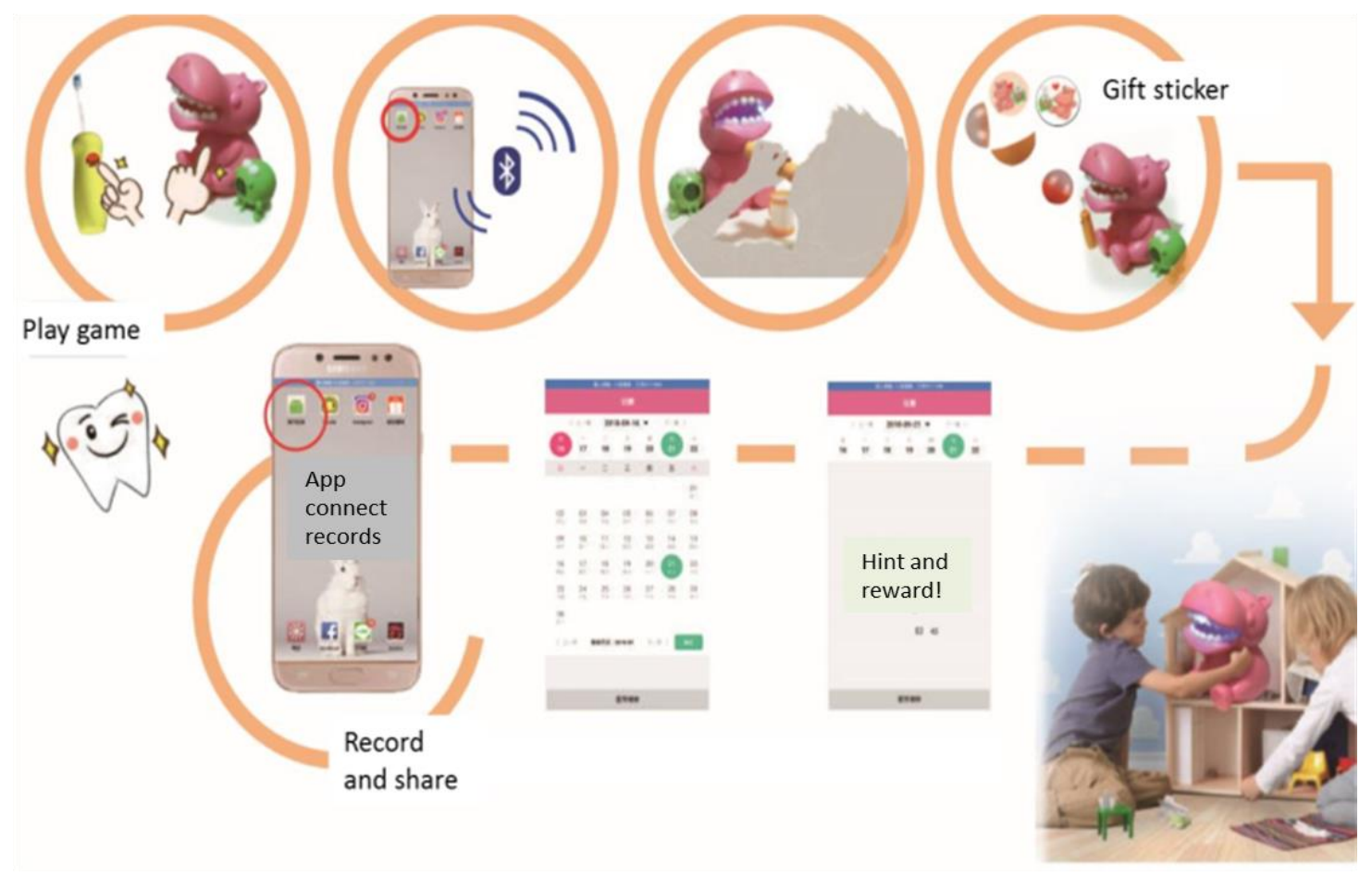

Figure 13. Operation flow and parent APP interface screen diagram.

The module comprised of a 3-watt speaker and a 3.7V lithium batteries. A brush circuit board contained the Power Module (Adio-5VCH + ADIO-USB-0506 Boost Module), Arduino IMU inertial sensor, and MPU-6050. An indicator interface placed inside each tooth, which lit up to indicate cleaning progress. The current installation appearance, and future possible grip shape are display in Figure 12.

\subsection{User Interface. Design}

The product placed in homes or classrooms. The intended users were children who had just begun to clean their teeth. Parents or teachers could use the game machine to demonstrate brushing to children.

Children have their feelings and tastes; designers of new technologies need to remind that children are an entirely different user. Researchers suggested "children had more direct involvement with technology developers" for technology development and invited children to test before it released [14]. The interactive experience is the basis for the toy design. Lacking researches on children's interactive experience causes mismatching of children's needs [15]. Popular toys are a dominant entertainment medium. Traditional toys have the function of supporting children's growth [16]. The user interface of a childfriendly product is one of the elements of a successful design.

The Figure 13 shows the operation process and parent's APP interface. This app can record the score and progressing of cleaning events, gift sticker reward, and parent responses. A monthly calendar is helpful to view children's oral cleaning status and progress. Designers are required to avoid using symbolic images that confuse children. The contrast formed by different color blocks is natural to arouse the user's visual interest. Deleting extra pages simplifies the process and only shows what is needed to convey important messages - the interface mainly based on natural and pleasant feelings, and brighter colors matched with cute fonts.

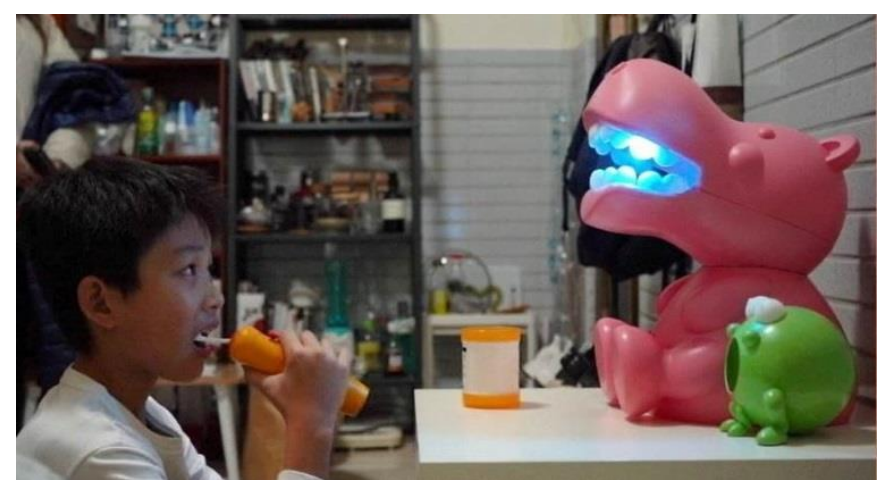

Figure 14. Product usage test: after the sensor has been swiped for a set number of times, the indicators will jump in the sequence. The reward gift is placed in the mouth of the villain on the lower right.

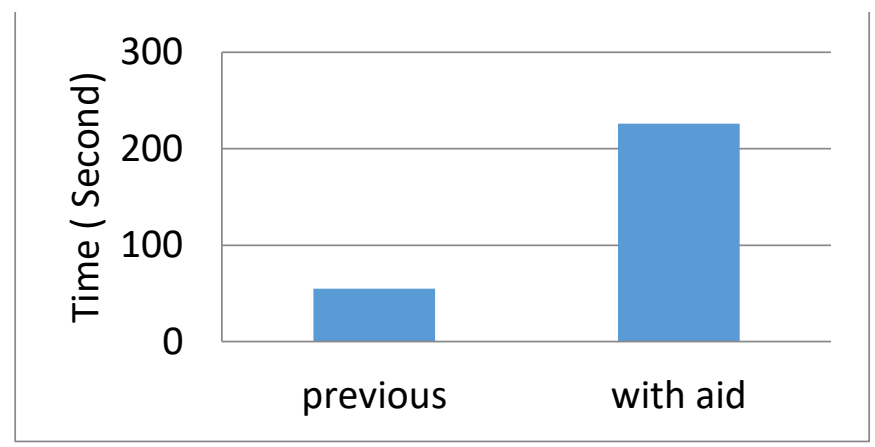

Figure 15. Product usage test: the average brushing time

\subsection{User's Test}

The product usage test carried on in both family house and kindergarten classrooms (Figure 14). The researcher invited five children to complete the brushing steps according to the light of assisting partners and record children's responses and the total time of brushing. From the experiment, we observed that children 


\section{Chuko et al. / Advances in Science, Technology and Engineering Systems Journal Vol. 5, No. 2, 494-499 (2020)}

became more attentive and adjusted to their corresponding tooth positions according to changes in the hippo's dental light. Figure 15 is the average brushing time result; the autonomous brushing is often completed quickly (55 seconds) with the disadvantage of less fulfilling the requirements. But the average time for brushing increased to 226 seconds with the assistance of the hippo partner. The teacher's required value is 180 seconds ( $3 \mathrm{~min}$.) [2], and the average acting time is higher. During the boot test, the child must wait for the light to change position (time delay), so it will take more time to follow at the beginning. The classroom setting also takes advantage of the children's inclination to increase enthusiasm within a peer-collective situation [3].

Children were happy to follow the LED display of the hippo and complete the steps of brushing their teeth through interaction. When parents need to accompany them, the child will also be stressed; parents' time is limited and it is not easy to maintain good mood. Through the records left by the interaction with the companion, parents can reward and respond to the child after watching it. This approach reduces tension and wasted time on both sides.

However, the mechanism of this model encountered some configuration problems. The brush's rod is insufficiently supported, so when the child brushes his teeth, the head is biased; water may flow along the toothbrush rod into the circuit area in the handle. The added electronic induction circuit does increase the issue of safety and must be waterproof and damp-proof before entering the application. At present, the size of the product is large, and the toothbrush grip also weighs a significant amount. The component and device size can reduce in the future with the help of electrical and mechanical engineers in the cross-domain design.

\section{Conclusions}

Throughout the investigation, the researchers identified the current educational need for dental care. An interactive novelty toothbrush with Bluetooth module was built and evaluated. Building cross-domain design teams allowed conceptual revision and determination in the early stages of design. Through cooperation with electrical engineers and circuit implementation of Arduino, design elements were combined. The children connected with parents through the app interface. This design can reduce the burden of parental supervision and contribute to the positive cycle of child interaction. At present, the cost of firmware hardware is high, so it is more suitable for teaching aids. In the future, the miniaturization of electronic parts will make the product more suitable for children.

\section{Conflict of Interest}

The authors declare no conflict of interest.

\section{Acknowledgment}

The authors acknowledge the electrical engineers in cross-domain design and programming.

\section{References}

[1] https://www.buysplat.com/pages/bass-brushing-technique

[2] M. Poyato-Ferrera, J. J. Segura-Egea, and P. Bullón-Fernández. "Comparison of modified Bass technique with normal toothbrushing practices for efficacy in supragingival plaque removal" International Journal of Dental Hygiene, 1(2), 110-114, 2003. https://doi.org/10.1034/j.16015037.2003.00018.x
[3] E. Robinson, "A comparative evaluation of the Scrub and Bass Methods of toothbrushing with flossing as an adjunct (in fifth and sixth graders)." American Journal of Public Health, 66(11), 1078-1081, 1976. https://ajph.aphapublications.org/doi/pdf/10.2105/AJPH.66.11.1078

[4] S.P Patil, P.B. Patil, and M.V. Kashetty. "Effectiveness of different tooth brushing techniques on the removal of dental plaque in 6-8-year-old children of Gulbarga." Journal of International Society of Preventive \& Community Dentistry, 4(2), 113, 2014. https://doi: 10.4103/2231-0762.138305

[5] P.R. Warren, M.A. Cugini, B.V. Chater, and J. Strate, "A review of the clinical efficacy of the Oral-B oscillating/rotating power toothbrush and the Philips Sonicare toothbrush in normal subject populations" International Dental Journal, 54(6), 429-437, 2004. https://doi.org/10.1111/j.1875595X.2004.tb00300.x

[6] T. J. Holley, J. A. Ross, and T. L. Hottel, "Efficacy of Philips Sonicare AirFloss compared to manual brushing and flossing: an in vitro study" The Journal of the Tennessee Dental Association 94(1), 34-36, 2014. https://europepmc.org/article/med/25241500

[7] G.I. McCracken, P.M. Preshaw, L. Heasman, "Efficacy of plaque removal of the Sonicare Elite versus the Sonicare Advance from hard-to-reach sites" Journal of Clinical Periodontology. Nov;31(11):1007-1011, 2004. DOI: 10.1111/j.1600-051x.2004.00604.x.

[8] B. Tiffany, P. Blasi, S.L. Catz, and J.B. McClure, "Mobile apps for oral health promotion: Content review and heuristic usability analysis" JMIR mHealth and uHealth, 6(9), p.e11432., 2018.

[9] P. Nayak, S. Nayak, M. Vikneshan, S. Acharya, and D. Sathiyabalan, "Smartphone apps: A state-of-the-art approach for oral health education" Journal of Oral Research, 8(5), 386-393, 2020. https://doi.org/10.17126/10.17126/joralres.

[10] F. Zotti, D. Dalessandri, S. Salgarello, M. Piancino, S. Bonetti, L. Visconti, C. Paganelli, "Usefulness of an App in improving oral hygiene compliance in adolescent orthodontic patients" The Angle Orthodontist, (1), 101-107, 2016. https://doi.org/10.2319/010915-19.1

[11] J. Blum, Exploring Arduino: Tools and techniques for engineering wizardry, John Wiley \& Sons, 2019.

[12] N. Cameron, 4-Digit 7-Segment Display, Arduino Applied. Apress, Berkeley, CA, 119-136, 2019.

[13] A. Verma, G. Culbertson, and K. Ramani, "Chiron: interpreting signals from capacitive patterns and inertial sensors for intuitive shape modeling" CHI'14 Extended Abstracts on Human Factors in Computing Systems, 1831-1836, 2014. https://doi.org/10.1145/2559206.2581161

[14] A. Druin, "The role of children in the design of new technology." Behaviour and information technology, 21(1), 1-25, 2002.

[15] X. Lv \& R. Peng, "User experience research of toy design" In IEEE 11th International Conference on Computer-Aided Industrial Design and Conceptual Design, CAID and CD'2010, 2, 943--946. DOI: 10.1109/CAIDCD.2010.5681811

[16] A.A. Naufal, and A. Suzianti, "Design thinking approach for product innovation design of educational toys"In Proceedings of the 20195 th International Conference on E-business and Mobile Commerce, 69-74, 2019. https://doi.org/10.1145/3332324.3332337 